\title{
Perfis de jogadores em contextos de ensino/aprendizagem em disciplinas de programação
}

\author{
José Arnóbio de Oliveira Júnior ${ }^{1}$, Alexandre A. Barbosa ${ }^{1,2}$ \\ ${ }^{1}$ Universidade Federal de Alagoas - Campus Arapiraca (UFAL) \\ Caixa Postal 61 - Arapiraca - AL - Brasil \\ ${ }^{2}$ Departamento de Sistemas e Computação \\ Universidade Federal de Campina Grande (UFCG) \\ Caixa Postal 10.106 - Campina Grande - PB - Brasil \\ arnobiojroliveira@gmail.com, alexandre.barbosa@arapiraca.ufal.br
}

\begin{abstract}
We can easily find in the literature, researches that investigates if the use, or the development of games, in educational contexts can generate benefits for learning activities. In addition, is also becoming quite common the gamification of learning environments. In this research, we present an experiment conducted in order to verify if a gamer type can be used as a strategy to group users/students in educational contexts. A gamer type may be described as the way that an individual relates to the game. In the experiment questionnaires were used to identify the gamer types, at one of the questionnaires the questions describe situations in a game context and in the other the questions describes an educational environment. The identified gamer types were compared using as a metric the Cohen's kappa. The results showed a substantial agreenment (Kappa $=0.657$ ) between the classifications. As a contribution, given the results, it is suggested that a gamer type can be used in educational settings to optimize environments for each different profile.
\end{abstract}

Resumo. Podem ser encontrados facilmente na literatura trabalhos que investigam se o uso ou a construção de jogos em contextos educacionais gera benefícios ao aprendizado. Além destes, também vem se tornando bastante comum a Gamificação de ambientes de ensino. Neste artigo, apresentamos um experimento realizado com o intuito de verificar se perfis de jogadores podem ser utilizados como meio para agrupar usuários/alunos em contextos educacionais. Um perfil de jogador pode ser descrito como a maneira que um indivíduo se relaciona com o jogo. No experimento realizado foram utilizados questionários para identificação de perfis, sendo um destes um ambiente descrito como um jogo e outro em um ambiente educacional. Os perfis identificados foram então comparados utilizando como métrica o Kappa de Cohen. Os resultados obtidos demonstraram uma concordância substancial (Kappa $=0.657$ ) entre as classificações obtidas. Como contribuição, dados os resultados, é sugerido que um perfil de jogador possa ser utilizado em ambientes educacionais visando otimizar os ambientes para cada diferente perfil de jogador.

\section{Introdução}

Muitas pesquisas buscam entender definições e motivos pelos quais as pessoas jogam determinado jogo, alguns destes estudos, tais como [Bartle 1996], [Nacke et al. 2011] e 
[Marczewski 2015], são focados no entendimento dos diferentes perfis de jogadores. Um perfil de jogador pode ser descrito como a maneira com a qual um indivíduo se relaciona com o jogo. Assim, vários autores interessados no tema analisam o que cada indivíduo possui como motivação e o que necessitam como estímulos específicos para serem sensibilizados por um jogo. Estes estudos também são fomentados pela necessidade de desenvolvimento de jogos cada vez mais adequados a maneira como um usuário se relaciona ao ambiente.

Realizando uma pesquisa apenas nos anais do Workshop de Ensino em Pensamento Computacional, Algoritmos e Programação do ano de 2015, é possível encontrar 6 trabalhos (16\% dos trabalhos publicados), tais como, [Aguiar 2015] e [Raabe et al. 2015], relacionados ao uso de jogos ou a gamificação em ambientes de ensino. Desta forma, observando o crescente interesse em pesquisas relacionadas ao uso de jogos na área de educação, nos motivou a formulação da seguinte questão de pesquisa "um perfil de jogador pode ser utilizado em um ambiente educacional?". Caso um perfil de jogador seja adequado para estes ambientes, todo o esforço da área de identificação de perfis de jogadores pode ser transportado para contextos educacionais.

Neste trabalho é apresentado um experimento que consistiu em comparar se um aluno possui um mesmo perfil tanto em um jogo quanto em um ambiente educacional. O modelo de perfis de jogadores utilizado é o modelo proposto por Richard Bartle [Bartle 1996], onde estão presentes os perfis Conquistadores (Achiever), Exploradores (Explorer), Assassinos (Killer) e Socializadores (Socializer). Para realizar a identificação dos perfis dos alunos foram utilizados questionários com perguntas de múltipla escolha.

Este trabalho está estruturado da seguinte maneira, na seção 2 são apresentados os modelos de Bartle, sendo este conhecimento necessário para compreensão da temática desta pesquisa. Na seção 3, são apresentados os trabalhos relacionados aos temas de modelagem de perfis de jogadores e identificação, ou classificação, de jogadores/alunos. Na seção 4 são apresentados os detalhes da metodologia e condução desta pesquisa. Finalmente na seção 5 são descritas as conclusões e trabalhos futuros.

\section{Fundamentação teórica}

\subsection{Modelo de Bartle (Arquétipos de Bartle)}

Richard Bartle foi o criador do primeiro Multi User Dungeon (MUD), gênero de videogames no qual os jogadores desempenhavam o papel de um personagem em um cenário fictício, com mundos virtuais multiplayer. Neste jogo, a interação ocorria em modo texto, ou seja, havia apenas um tipo de tela de bate-papo sem nenhum outro recurso gráfico onde os jogadores digitavam suas ações e o jogo retornava as reações correspondentes. Hoje, em um cenário completamente diferente e evoluído, estes jogos são chamados de Massive Multiplayer Online Game ou MMORPG.

Com o pioneirismo e interesse na área, Bartle desenvolveu um modelo de perfis de jogadores conhecido como arquétipos de Bartle (ou modelo de Bartle) [Bartle 1996]. Neste trabalho, Bartle descreve um estudo baseado nos comportamentos e objetivos alcançados pelos jogadores em um jogo do tipo MUD. Tal estudo identificou 4 perfis de jogadores: Conquistadores (Achiever), Exploradores (Explorer), Assassinos (Killer) e Socializadores (Socializer). 
Cada perfil se comporta de uma maneira diferenciada e é motivado por determinados elementos e ações no cenário do jogo. Os Socializadores são mais atraídos pelo relacionamento com outros jogadores, no que o outro tem a dizer, mesmo que isso não faça tanta diferença para o seu desenvolvimento durante o jogo. O perfil que se preocupa principalmente em eliminar todos os adversários é o Assassino. Os Assassinos quase sempre demonstram espírito de liderança e gostam de semear a destruição. Já os Exploradores gostam de descobrir tudo relacionado aos aspectos do cenário, conhecer e entender o máximo possível do jogo. Por fim, os Conquistadores são movidos pelos objetivos do jogo ou qualquer maneira de acumular pontos, experiência, níveis, troféus, tudo o que destaque-o em relação aos outros jogadores.

A Figura 1(a) exemplifica como Bartle classificou os perfis quanto às aspirações. São traçados dois eixos que definem qual quadrante pertence o indivíduo. Horizontalmente são colocados as vertentes relativas a outros jogadores e mundo (cenários, objetos, etc). Verticalmente são observadas as vertentes 'agindo' e 'interagindo'. Assim, um jogador classificado no modelo de Bartle será dito como: Agindo sobre os jogadores, Interagindo sobre o mundo, e assim sucessivamente.

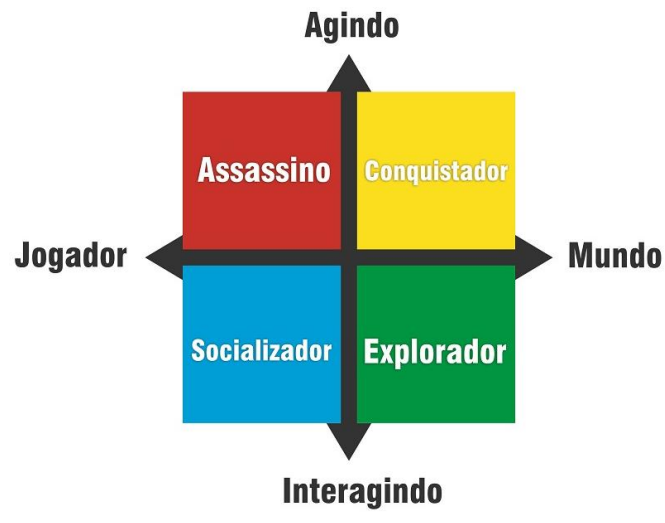

(a) Modelo original de Bartle.

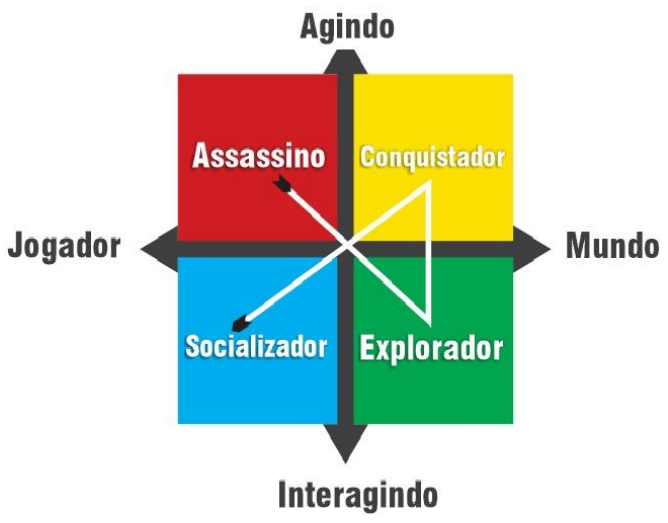

(b) Ciclo de mudanças descrito no modelo revisado de Bartle.

Figura 1. Arquétipos de Bartle e ciclo de mudanças

Em [Bartle 2005], Bartle revisou sua proposta original de classificação de perfis de jogadores. Nesta revisão, foi discutido que embora o modelo tenha sido utilizado por várias pessoas, existiam algumas limitações no mesmo. Nesta nova proposta, é sugerido que os jogadores podem mudar de perfil com o passar do tempo, mas não é proposto como ou porque eles podem chegar a fazer isso. Em segundo lugar, é perceptível que todos os perfis, em determinadas proporções, demonstram ter perfis paralelos que o modelo original não predizia.

As limitações identificadas foram resolvidas com a adição das variáveis implícito e explícito. Uma ação implícita é aquela que acontece automaticamente, sem intervenção da mente consciente. Já a ação dita explícita é geralmente planejada, considerada como uma maneira de conseguir algum objetivo ou efeito. Bartle deixa claro que este novo modelo explica as diferenças entre os subtipos de perfis, mas não justifica o real motivo pelo qual os jogadores realizam trocas entre os perfis ao longo do tempo.

A mudança de perfil mais comum foi Assassino para Explorador para Conquis- 
V Congresso Brasileiro de Informática na Educação (CBIE 2016)

Anais dos Workshops do V Congresso Brasileiro de Informática na Educação (CBIE 2016)

tador para Socializador, como ilustrado na Figura 1(b). Por ter sido a mais popular, esta sucessão foi denominada 'Sequência Principal'.

A representação gráfica da ideia original foi feita em um plano de duas dimensões, relacionando interação (agindo e interagindo) e com quem é realizada esta interação (jogadores e mundo). Já para o novo modelo, com a adição de outra dimensão, foi necessário uma figura em 3 dimensões para representar bem cada um dos novos perfis. A representação do modelo revisado pode ser observada na Figura 2.

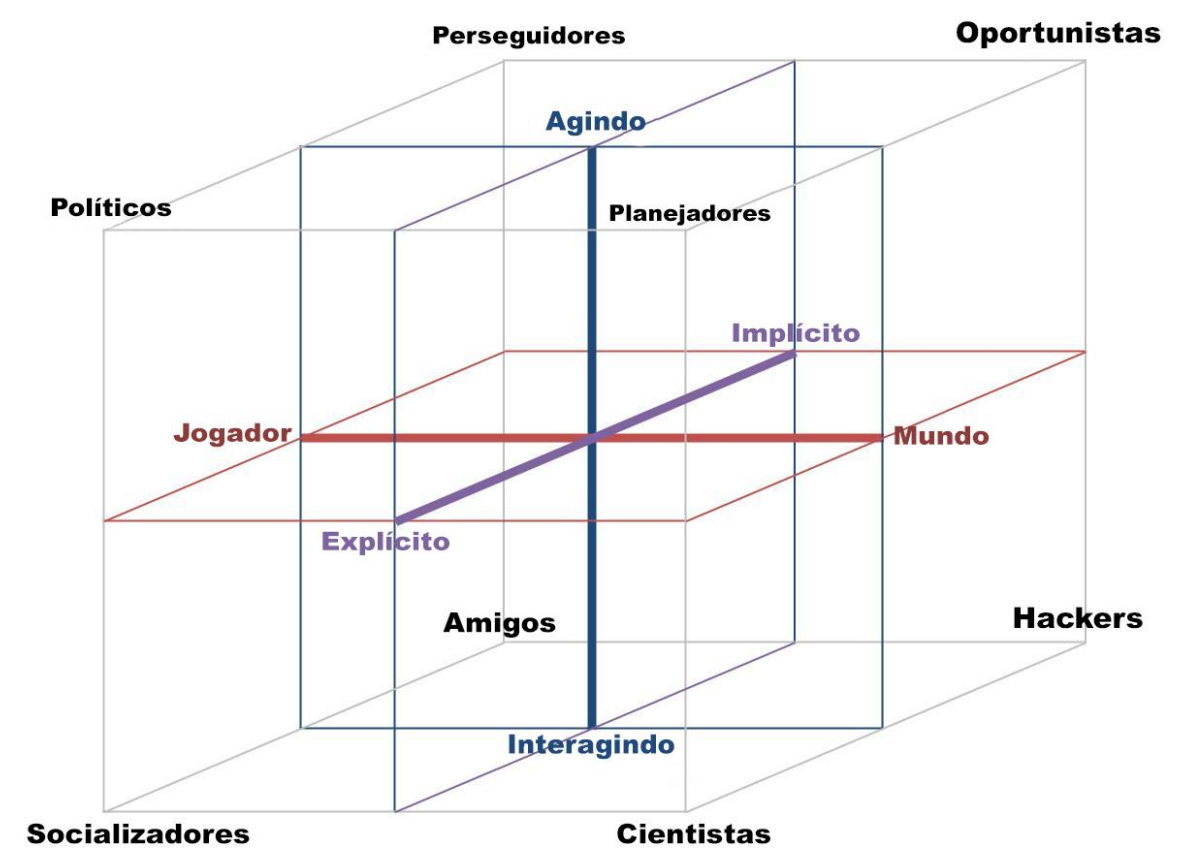

Figura 2. Modelo revisado de Bartle. Adaptado de [Nacke ]

Desta forma, ao invés de 4, existem 8 perfis de jogadores observados. São eles:

1. Oportunistas/Opportunists (Conquistadores implícitos) - aproveitam todas as oportunidades que se apresentam;

2. Planejadores/Planners (Conquistadores explícitos) - focam em um objetivo e se destinam a alcançá-lo;

3. Cientistas/Scientists (Exploradores explícitos) - experimentam para teorizar;

4. Hackers (Exploradores implícitos) - experimentam para revelar o significado, descobrir novos fenômenos;

5. Socializadores/Networkers (Socializadores explícitos) - procuram interação com pessoas;

6. Amigos/Friends (Socializadores implícitos) - interagem com pessoas conhecidas;

7. Perseguidor/Griefers (Assassinos implícitos) - muito competitivos e agressivos;

8. Políticos/Politicians (Assassinos explícitos) - manipulam as pessoas sutilmente, agindo com prudência e previsão.

\subsection{Kappa de Cohen}

Para saber se uma dada caracterização ou classificação de uma instância é confiável, é necessário ter esta instância caracterizada ou classificada várias vezes. De posse deste 
conjunto de classificações, o Kappa de Cohen é utilizado para medir a intensidade da concordância entre dois ou mais avaliadores, ou entre dois métodos de classificação.

É importante frisar que este critério de concordância mede o grau de concordância além do que seria esperado tão somente pelo acaso. O coeficiente Kappa de Cohen é dado por $K a p p a=\frac{P_{o}-P_{c}}{P-P_{c}}$, onde $P_{o}$ é a proporção de observações, $P_{c}$ é a proporção de concordância devida ao acaso e $P$ é o número de instâncias. Os valores obtidos podem variar no intervalo $[-1,1]$. O resultado máximo 1 , representa total concordância, valores positivos indicam a intensidade de concordância entre os avaliadores, o valor 0 (zero) indica nenhuma concordância, ou que a concordância foi exatamente a esperada pelo acaso. Um eventual valor negativo, sugere que a concordância encontrada foi menor do que a esperada por acaso. Sugere, portanto, discordância, mas seu valor não tem interpretação como intensidade de discordância.

No intervalo positivo uma descrição da concordância pode ser fornecida:

- $0<$ kappa $<0.2$, concordância leve;

- $0.2 \leq$ kappa $<0.4$, concordância razoável;

- $0.4 \leq$ kappa $<0.6$, concordância moderada;

- $0.6 \leq$ kappa $<0.8$, concordância substancial;

- $0.8 \leq$ kappa $<1.0$, concordância quase perfeita;

- kappa $=1$, concordância perfeita;

\section{Trabalhos relacionados}

Visto que o presente trabalho tem como objetivo classificar sujeitos em um conjunto de classes correspondentes a perfis de jogadores. Nesta seção são apresentados os trabalhos relacionados divididos em dois temas: os trabalhos que tratam da modelagem de perfis de jogadores, exibidos na seção 3.1; e outros que buscam desenvolver ou validar mecanismos para classificar um sujeito em um determinado perfil/classe, sendo estes descritos na seção 3.2 .

\subsection{Modelos de perfis de jogadores}

No trabalho de Nick Yee [Yee 2005], baseado no modelo original de Bartle, o autor teve como objetivo a reorganização da relação entre perfis de jogadores com situações reais de jogo e interesses psicológicos. A divisão de perfis proposta no trabalho engloba três grupos, sendo estes: Conquistas (Achievement), Sociabilidade (Social) e Imerssão (Immersion). Cada grupo é motivado por determinadas tendências, organizadas pelos subcomponentes. O perfil Achievement, por exemplo, é inclinado a realizar conquistas, adquirir poder e otimizar seu avatar para competir e desafiar os outros jogadores. O perfil Social busca relacionar-se de forma que possa ajudar e conversar com os outros, seus objetivos são interagir e colaborar com todos os outros participantes. Finalmente o perfil Immersion busca descobrir e conhecer características do jogo/mundo que a maioria dos outros jogadores não conhece.

Marczewski apresenta em [Marczewski 2015] um modelo, também baseado no modelo original de Bartle, onde os perfis são classificados quanto ao tipo de recompensa pelo qual se interessam. Neste modelo existem seis tipos básicos de usuários, sendo estes divididos em dois grupos, os usuários intrínsecos, Conquistador (Achiever), Socializador (Socialiser), Filantropo (Philanthropist) e Espíritos Livres (Free Spirit), e os usuários 
extrínsecos, Disruptor Disruptor e Jogador (Player). O grupo de usuários extrínsecos possui motivação baseada em fatores externos ao indivíduo, como qualquer recompensa em dinheiro ou riqueza, o jogador executa as ações para ser recompensado ou para não ser castigado. O grupo de usuários intrínsecos possui motivação baseada em fatores internos ao indivíduo, esta muito mais ligada com a sua forma de ser, os seus interesses e preferências, não há necessidade de recompensar o jogador, pois ele realiza as ações por estas serem algo que ele gosta.

O estudo conduzido em [Drachen et al. 2009] analisou a forma como um conjunto de jogadores completaram o jogo de aventura Tomb Raider: Underworld. Foram caracterizados quatro estilos diferentes de jogadores, cada um com diferentes padrões de jogo e soluções para problemas específicos e desempenho. Para análise foram utilizadas informações do registro de jogo como tempo de conclusão, número de mortes, causas de morte e ajuda em ações. Os perfis identificados foram: Veteranos (Veterans), são os jogadores mais experientes, morrem muito raramente e completam o jogo muito rápido; Solucionadores (Solvers) gastam seu tempo para resolver os enigmas encontrados durante o jogo; Pacifistas (Pacifists) são aqueles que são mortos quase sempre por inimigos ou obstáculos e são razoavelmente rápidos quanto a completar o jogo; Corredores (Runners) recebem este nome pela rápida realização do jogo, porém morrem muitas vezes e recorrem a um número considerável de ajudas.

Dentre os vários modelos de perfis de jogadores propostos, é possível notar a grande influência do modelo original de Bartle. Desta forma, mesmo tendo esse modelo sofrido uma revisão, justificamos a escolha do modelo original para a realização do experimento, pois espera-se conseguir uma maior generalização para os resultados.

\subsection{Classificação/Identificação de perfis}

A classificação de perfis é um tema estudado em diversas áreas e quase sempre tem como objetivo a personalização de algum conteúdo para o usuário ou análise de determinados grupos. No contexto de ambientes educacionais, em geral, os usuários são classificados de acordo com estilos de aprendizagem. A seguir são descritos alguns trabalhos que buscam identificar o perfil de um usuário e fornecer algum retorno adequado a este perfil.

Em [Oliveira et al. 2014], perfis de aprendizagem na prática de programação foram identificados a partir de análise fatorial de componentes latentes que caracterizem os desempenhos de uma turma em atividades. Aliado a esta técnica, clusters foram utilizados para agrupamentos de perfis similares. O objetivo dessa metodologia é mostrar como a combinação das técnicas de análise fatorial e de clustering pode melhorar o processo de classificação de perfis. Os primeiros resultados indicaram que essa estratégia pode ser aplicada para melhor selecionar amostras de perfis.

O trabalho de [Bativa and Stiubiener 2011] buscou analisar algumas das principais teorias e metodologias para a estimativa de perfis de aprendizes e apresentar uma ferramenta de identificação de perfis desta área. Os estilos de aprendizagem baseiam-se em dois eixos: a captação - exercitar a percepção, decodificando as informações externas e aproximando-as da sua vivência - e a transformação da informação - significância da informação através da reflexão e análise crítica, o que leva a uma nova organização das mesmas. A Ferramenta de Identificação de Perfis de Aprendizes (FIPA), foi fundamentada nos procedimentos de estilos de aprendizagem de pesquisadores da área, relaci- 
onados a componentes intelectuais pessoais de assimilação de informação e componentes estáveis, relativos a fatores cognitivos da personalidade. Este mecanismo permite que, por exemplo, seja preparado um material didático para um melhor aproveitamento da classe e propiciar ao aluno maior eficácia no seu aprendizado.

De forma totalmente transparente e implícita ao usuário, o estudo de [Rabelo et al. 2010] buscou identificar e extrair, por meio da técnica de inteligências múltiplas, perfis individuais intelectuais durante a utilização de um ambiente virtual de aprendizagem (AVA) desenvolvido para o Projeto EDULIVRE (Tecnologias Educacionais Criativas Livres). Foi implementado no sistema uma funcionalidade que identificasse potencialidades especificas, dificuldades e inclinações cognitivas dos alunos. Para tal, a metodologia foi dividida nas seguintes etapas: (1) Definir os conteúdos, (2) Produzir ou selecionar os objetos de aprendizagem, (3) Incluir os objetos de aprendizagem no repositório de dados, (4) Classificar de acordo com as inteligências múltiplas, (5) Monitorar utilização para traçar o perfil intelectual individual e (6) Exibir o perfil intelectual individual do educando. A contribuição foi dita relevante, uma vez que o educador passa a ter à sua disposição um importante instrumento de apoio no acompanhamento do processo ensino-aprendizagem ao longo de todo período.

\section{Metodologia, resultados e discussão}

Para condução do experimento foram utilizados dois questionários. O primeiro questionário, que chamaremos de questionário de Bartle, visava capturar o perfil de jogador de acordo com o modelo de Bartle, para isso foram utilizadas 30 perguntas do questionário disponibilizado em [GamerDNA ]. Para o segundo questionário, que chamaremos de questionário de programação, o objetivo era identificar um perfil equivalente ao modelo de Bartle, mas neste caso para um contexto educacional no âmbito de programação. Os questionários utilizados estão disponíveis em um formulário online ${ }^{1}$ os questionários são aplicados em sequência.

Os dados do experimento foram coletados a partir de 15 sujeitos, sendo 14 do sexo masculino e 1 do sexo feminino, todos declararam gostar de jogos, em sua maioria do estilo Role Playing Game (RPG) ou MMORPG. Todos os sujeitos eram alunos do curso de computação de uma universidade federal.

As perguntas do questionário de Bartle possuíam duas alternativas, cada uma destas correspondia a uma proposição voltada a um dos quatro perfis. Neste o respondente deveria escolher uma das duas alternativas apresentadas. Tal como exibido nos exemplos a seguir:

1. Em um $M M O R P G$, surge uma nova area. O que você mais ansiosamente espera?

(a) Ser o primeiro a ter o equipamento da área (Achiever)

(b) Explorar a nova área, descobrindo sua história (Explorer)

2. Você está prestes a entrar em um calabouço desconhecido. Você tem a escolha de mais uma pessoa para a sua caminhada

(a) Um bardo, que é um bom amigo de vocês e que é ótimo para entreter você (Socializer)

\footnotetext{
${ }^{1}$ https://docs.google.com/forms/d/1y3NAefii9G91YQfqKqjATJjdMDElkyw9X2n8dXlfuz4/ viewform
} 
V Congresso Brasileiro de Informática na Educação (CBIE 2016)

Anais dos Workshops do V Congresso Brasileiro de Informática na Educação (CBIE 2016)

(b) Um bruxo, para identificar os itens que você encontrar lá (Explorer)

Visando minimizar a quantidade de perguntas necessárias para identificação do perfil, no questionário de programação eram apresentadas quatro alternativas de resposta, sendo cada uma voltada a um perfil. Neste o respondente deveria fornecer um valor, entre 1 e 4, para cada alternativa, sendo (1) a alternativa com a qual mais se identificava e (4) a alternativa qual menos se identificava. Tal como exibido nos exemplos a seguir:

1. Quais competências considera mais importantes para um programador:

(a) dominar profundamente uma linguagem de programação (Killer)

(b) obter certificação em uma linguagem de programação (Achiever)

(c) conhecer diversas linguagens (Explorer)

(d) participar ativamente da comunidade de desenvolvedores (Socializer)

2. Nas aulas de programação, você prefere

(a) Derrotar seus colegas em disputas (Killer)

(b) Ir ganhando experiência mais rápido que qualquer um (Achiever)

(c) Resolver um problema que ninguém tenha conseguido (Explorer)

(d) Convencer alguém para te ajudar com os problemas (Socializer)

O resultado para ambos os questionários correspondia a uma lista com o percentual de cada perfil que o respondente possuía. O resultado de um dos sujeitos do experimento é apresentado como exemplo na Tabela 1.

Tabela 1. Resultado dos perfis identificados para um sujeito do experimento

\begin{tabular}{|c|c|c|c|c|}
\hline & Achiever & Explorer & Killer & Socializer \\
\hline \hline Bartle & $34 \%$ & $66 \%$ & $53 \%$ & $47 \%$ \\
\hline Programação & $38 \%$ & $90 \%$ & $24 \%$ & $48 \%$ \\
\hline
\end{tabular}

Como é possível observar a soma dos percentuais gera um resultado de $200 \%$, sendo esse método de cálculo adotado no questionário original disponibilizado em [GamerDNA ]. Os questionários utilizados no experimento mantiveram o mesmo método de cálculo.

O resultado fornecido pelo questionário permite enumerar o quanto de cada perfil um sujeito possui. Contudo, nesta pesquisa a comparação dos perfis foi realizada somente com o principal perfil identificado. No caso do exemplo exibido anteriormente, em ambos o perfil identificado como principal foi Explorer. Na Tabela 2 é exibida a lista dos perfis principais identificados para cada um dos sujeitos que participaram com o uso dos dois questionários.

A lista de perfis obtida em cada um dos questionários foi então comparada utilizando como métrica o Kappa de Cohen. Os resultados obtidos demonstraram uma concordância substancial ( Kappa $=0.657$ ) entre as classificações obtidas. Sendo assim, sugere-se que um perfil de jogador pode ser utilizado em um ambiente educacional.

Contudo, vale salientar uma pequena quantidade de sujeitos foi utilizada no experimento, todos estes sujeitos possuíam perfis similares, ou seja estudantes universitários, que gostam de jogos e preferem o estilo de jogo RPG/MMORPG. Sendo o modelo de Bartle proposto para $M U D s$, é possível que para sujeitos com perfis diferentes, ou seja, 
V Congresso Brasileiro de Informática na Educação (CBIE 2016)

Anais dos Workshops do V Congresso Brasileiro de Informática na Educação (CBIE 2016)

Tabela 2. Lista dos perfis identificados

\begin{tabular}{|c|c|c|}
\hline Sujeito & Perfil Bartle & Perfil Programação \\
\hline \hline 1 & Explorer & Killer \\
\hline 2 & Explorer & Explorer \\
\hline 3 & Explorer & Killer \\
\hline 4 & Explorer & Explorer \\
\hline 5 & Killer & Explorer \\
\hline 6 & Explorer & Killer \\
\hline 7 & Achiever/Killer & Explorer \\
\hline 8 & Achiever & Achiever \\
\hline 9 & Socializer & Socializer \\
\hline 10 & Achiever & Achiever \\
\hline 11 & Explorer & Explorer \\
\hline 12 & Killer & Socializer \\
\hline 13 & Socializer & Explorer \\
\hline 14 & Explorer & Explorer \\
\hline 15 & Socializer & Explorer \\
\hline
\end{tabular}

pessoas que não gostem de jogos, ou que prefiram um estilo de jogo diferente, os resultados obtidos não corroborem com aqueles aqui exibidos.

Na seção 3 foram descritos brevemente diversos modelos de perfis de jogadores. No presente trabalho foi realizada a comparação da identificação de perfis apenas com um dos modelos existentes e descritos na literatura, o modelo original proposto por Richard Bartle. É possível que com o uso de outros modelos de perfis de jogadores, tais como [Marczewski 2015] ou [Drachen et al. 2009], os resultados sejam diferentes daqueles aqui exibidos.

\subsection{Ameaças à validade}

As seguintes ameaças à validade estão presentes nesta pesquisa:

- Baixo poder estatístico - uma vez que apenas 15 sujeitos se apresentaram para realização da coleta de dados, é possível que os resultados obtidos não sejam representativos de um universo maior;

- Instrumento utilizado na classificação - o questionário utilizado para capturar o perfil dos jogadores pode não ser o meio mais adequado para tal ação, uma vez que o perfil de jogador é uma manifestação de características que ocorrem em um ambiente de jogo, este perfil pode ser melhor capturado fazendo uso de um jogo projetado para classificar seu comportamento.

\section{Conclusões e trabalhos futuros}

Neste artigo, apresentamos um experimento realizado para verificar se perfis de jogadores poderiam ser utilizados como meio para agrupar jogadores/alunos em contextos educacionais. Os resultados obtidos demonstraram uma concordância substancial (Kappa = 0.657) entre as classificações obtidas utilizando os questionários de identificação de perfis. Sendo assim, sugere-se que um perfil de jogador pode ser utilizado em um ambiente 
V Congresso Brasileiro de Informática na Educação (CBIE 2016)

Anais dos Workshops do V Congresso Brasileiro de Informática na Educação (CBIE 2016)

educacional. Em geral o intuito é explorar as características do perfil de jogador para otimizar o ambiente educacional para o aluno. Contudo, descrevemos que são necessários experimentos com uma quantidade maior de sujeitos e utilizando diferentes meios de identificação de perfis, para verificar se os resultados destes novos experimentos corroboram ou não com os resultados aqui apresentados.

\section{Referências}

Bartle, R. (1996). Hearts, clubs, diamonds, spades: Players who suit muds. The Journal of Virtual Environments, 1(1):19.

Bartle, R. (2005). Virtual worlds: Why people play. Massively multiplayer game development, 2(1):19.

Bativa, G. B. and Stiubiener, I. (2011). Ferramenta de identificação de perfis de aprendizes-fipa. In Anais do SBIE 2011.

Drachen, A., Canossa, A., and Yannakakis, G. N. (2009). Player modeling using selforganization in tomb raider: Underworld. In Computational Intelligence and Games, 2009. CIG 2009. IEEE Symposium on, pages 1-8. IEEE.

GamerDNA. Bartle test of gamer psychology. http://www.gamerdna.com/ quizzes/bartle-test-of-gamer-psychology. Ultimo acesso realizado em Junho de 2016.

Marczewski, A. (2015). User Types. In Even Ninja Monkeys Like to Play: Gamification, Game Thinking and Motivational Design. CreateSpace Independent Publishing Platform, 1st ed. edition.

Nacke, L. Dramatic elements of games and narrative design. http://www.acagamic.com/courses/infr1330-2014/

dramatic-elements-of-games-and-narrative-design/. Ultimo acesso realizado em Maio de 2016.

Nacke, L. E., Bateman, C., and Mandryk, R. L. (2011). Brainhex: Preliminary results from a neurobiological gamer typology survey. In Proceedings of the 10th International Conference on Entertainment Computing, ICEC'11, pages 288-293, Berlin, Heidelberg. Springer-Verlag.

Oliveira, M. G., Monroy, N. A. J., Zandonade, E., and Oliveira, E. (2014). Análise de componentes latentes da aprendizagem de programação para mapeamento e classificação de perfis. In Anais do SBIE 2014, page 134.

Raabe, A., Zanchett, G., and Vahldick, A. (2015). Jogos de programar como uma abordagem para os primeiros contatos dos estudantes com à programação. In Anais do WCBIE 2015.

Rabelo, H., Braga Jr, W. C., Borges, L. L., Bezerra, E. P., de Góes Brennand, E. G., Tavares, T. A., and de Souza Filho, G. L. (2010). Identificação do perfil individual intelectual do educando no ambiente virtual de aprendizagem edulivre. In Anais do SBIE 2010.

Yee, N. (2005). Motivations of play in mmorpgs. In International DiGRA Conference, Vancouver, British Columbia, Canada. 